nungsgemäß nennen, einen Link zur Creative Commons Lizenz beifügen und angeben, ob Änderungen vorgenommen wurden.

Die in diesem Artikel enthaltenen Bilder und sonstiges Drittmaterial unterliegen ebenfalls der genannten Creative Commons Lizenz, sofern sich aus der Abbildungslegende nichts anderes ergibt. Sofern das betreffende Material nicht unter der genannten Creative Commons Lizenz steht und die betreffende Handlung nicht nach gesetzlichen Vorschriften erlaubt ist, ist für die oben aufgeführten Weiterverwendungen des Materials die Einwilligung des jeweiligen Rechteinhabers einzuholen.

Weitere Details zur Lizenz entnehmen Sie bitte der Lizenzinformation auf http://creativecommons.org/ licenses/by/4.0/deed.de.

\title{
Schmied, Jürgen Peter (Hrsg.): Kriegerische Tauben. Liberale und linksliberale Interventionisten vom 19. Jahrhundert bis in die Gegenwart (Internationale Beziehungen. Theorie und Geschichte, Bd. 15), 206 S., V\&R unipress, Göttingen 2019.
}

\author{
Sebastian Elsbach \\ Online publiziert: 13 . Juli 2020 \\ (C) Der/die Autor(en) 2020
}

Der vorliegende Sammelband stellt die Frage, inwiefern liberale wie linksliberale Staatsmänner trotz wiederholter Bekenntnisse zu den Werten der Demokratie, des Friedens und der rechtsgebundenen Völkerverständigung zum Mittel des Krieges greifen, um ihre politischen Ansichten durchzusetzen. In acht Fallbeispielen werden die US-Amerikaner Thomas Jefferson, Woodrow Wilson, John F. Kennedy und Barack Obama, die Briten William E. Gladstone, David Lloyd George und Tony Blair, und (als einziger Deutscher im Bunde) Joschka Fischer behandelt. Die historische Bandbreite der Beiträge erstreckt sich somit auf drei Jahrhunderte, wie auch schon der Untertitel des Werkes ankündigt. Einleitend weist der Herausgeber Jürgen Peter Schmied darauf hin, dass ein Hauptargument liberaler Staatstheoretiker gegen den Monarchismus und für die republikanische Staatsform darin lag, dass letztere den Frieden begünstige, während Monarchen und andere autoritär Regierenden scheinbar skrupellos in den Krieg ziehen würden. So verweist Schmied auf das be-

\footnotetext{
S. Elsbach $(\bowtie)$

Friedrich-Schiller-Universität Jena, Jena, Deutschland

E-Mail: sebastian.elsbach@uni-jena.de
} 
rühmte Diktum Immanuel Kants aus „Zum ewigen Frieden“, wonach ein Zustand des Krieges gewissermaßen ,natürlich“ sei und der Frieden erst aktiv ,gestiftet“ werden müsse (S. 12). Auf Kant geht demnach auch die Theorie des Demokratischen Friedens zurück, also die Annahme, dass Demokratien untereinander keinen Krieg führen würden und auch insgesamt friedliebender seien als autoritär regierte Staaten (S. 12f.). Hinzu kommt die liberale Idee, dass der Freihandel den Frieden zwischen den Staaten stark begünstige, was eine wesentliche Legitimationsgrundlage für heutige internationale Organisationen wie die World Trade Organization oder auch die Europäische Union darstellt. Soweit die Theorie. Der Sammelband wagt gewissermaßen den Praxistest anhand der erwähnten Fallbeispiele. Eine Grundthese über „Liberale und Krieg“ benennt Dieter Langewiesche im ersten Beitrag: „Liberale Überzeugungen verpflichten nicht zum Pazifismus. [...] Im Kern beruhte diese [liberale] Bereitschaft zum Krieg auf der empirischen Beobachtung, die eigenen Ziele, von deren Fortschrittlichkeit man überzeugt war, würden sich anders nicht erreichen lassen“" (S. 25 ff.).

Die folgenden Beiträge des Bandes kreisen denn auch oft um den Nachweis, dass die jeweilige ,liberale Kriegsbereitschaft“ der Staatsmänner sich nicht mit einem Hinweis auf vermeintliche politische Sachzwänge, die ihre moralischen Grundwerte gewissermaßen überlagern und sie gegen ihren Willen in den Krieg treiben würden, erklären lasse. Im Gegenteil: Keiner der Staatsmänner sei in den Krieg ,gestolpert', sondern alle hätten (mehr oder weniger) klar umrissene Ziele umsetzen wollen. Dass diese Ziele immer ethisch vertretbar oder auch nur zweckrational gewesen seien, wird hierbei nicht behauptet. Während Langewiesche noch die Errichtung der europäischen Nationalstaaten im 19. Jahrhundert als grundsätzlich legitime liberale Zielsetzung aufzeigt und gleichzeitig festhält, die hierbei umgesetzte ,liberale Revolutionspolitik“ habe auf ein Minimalmaß an Gewalt abgezielt (S. 28ff.), stellt Jasper M. Trautsch im zweiten Beitrag über Jefferson fest, dass dessen Ziel einer US-amerikanischen Dominanz in Nordamerika ohne Kriege gegen die Europäer und die Unterdrückung von Ureinwohnern und Sklaven nicht realisierbar gewesen sei (S. 53 f.). Die Gleichzeitigkeit des „Bellizisten“ Jefferson und des „Pazifisten“ Jefferson erklärt Trautsch nicht als Gegensatz zur Theorie des liberalen Friedens, der auch Jefferson anhing, sondern als dessen Konsequenz, denn wo Demokratien und Monarchien nebeneinander existierten, sei Konflikt und letztlich auch der Krieg unvermeidlich. Oder um Jefferson zu zitieren: ,Wars then must sometimes be our lot“" (S. 54). Die christliche Unterfütterung der US-Interventionspolitik wird ebenfalls in den weiteren Kapiteln über US-Präsidenten behandelt, wobei dies noch am ehesten im Beitrag über Barack Obama, der offenbar ein Anhänger der chiliastischen Schriften Reinhold Niebuhrs ist (S. 179ff.), überrascht. Die Artikel zu Wilson und Kennedy legen denn auch ihren Schwerpunkt auf die machtpolitischen Kalküle der Präsidenten und nicht auf das besonders bei Wilson oft bemühte demokratisch-missionarische ,Kreuzritterdenken“ (S. 105). Kennedy gar wird ein geradezu zynischer Umgang mit seinen vietnamesischen Verbündeten attestiert und die Vorstellung, dass er die Intensivierung des Vietnamkrieges verhindert hätte, wenn er nicht ermordet worden wäre, als Legende entlarvt. Im Gegenteil habe gerade Kennedys Vietnampolitik die Grundlagen für die massive Gewalteskalation während der Präsidentschaft von Lyndon B. Johnson gelegt (S. 139f.). 
Aber auch die Beiträge über die europäischen Staatsmänner sind insofern lesenswert, als dass in ihnen versucht wird, so manch verbreitete Fehleinschätzung zu widerlegen. Etwas außenstehend in der Reihe englischsprachiger Staatsmänner ist gleichwohl Joschka Fischer, dessen Charakterisierung als Linksliberaler aber hinsichtlich seiner Wandlung in den 1990er Jahren stimmig ist. Fischer passt auch deshalb in die oben aufgezählte Reihe, weil an seinem Beispiel klar wird, wie prekär die liberalen Rechtfertigungsstrategien hinsichtlich einer Kriegs- und Interventionspolitik tatsächlich sind. Zur Legitimierung einer deutschen Beteiligung am Kosovokrieg bemühte Fischer medienwirksam den Vergleich mit ,Ausschwitz', dessen potenzielle Wiederholung auch mit dem Mittel des Krieges verhindert werden müsse (S. 152f.). Diese Verbindungslinie Fischers wurde vielfach als „Instrumentalisierung“ kritisiert und infolgedessen - wie Hans Kundnani prägnant schlussfolgert - ,scheint die Formel ,Nie wieder Krieg!‘ das Argument ,Nie wieder Auschwitz!“ als leitende[s] Prinzip der deutschen Außenpolitik wieder ersetzt zu haben" (S. 157).

Insgesamt liegt mit „Kriegerische Tauben“ ein meinungsstarker und daher anregender Sammelband vor, in dem lediglich ein Beitrag über den Zweiten Weltkrieg beziehungsweise Franklin D. Roosevelt zu vermissen ist, und der einen gelungenen Kontrapunkt zum Jahrbuch für Liberalismusforschung 2019 über „Liberale und Frieden" setzt.

Funding Open Access funding provided by Projekt DEAL.

Open Access Dieser Artikel wird unter der Creative Commons Namensnennung 4.0 International Lizenz veröffentlicht, welche die Nutzung, Vervielfältigung, Bearbeitung, Verbreitung und Wiedergabe in jeglichem Medium und Format erlaubt, sofern Sie den/die ursprünglichen Autor(en) und die Quelle ordnungsgemäß nennen, einen Link zur Creative Commons Lizenz beifügen und angeben, ob Änderungen vorgenommen wurden.

Die in diesem Artikel enthaltenen Bilder und sonstiges Drittmaterial unterliegen ebenfalls der genannten Creative Commons Lizenz, sofern sich aus der Abbildungslegende nichts anderes ergibt. Sofern das betreffende Material nicht unter der genannten Creative Commons Lizenz steht und die betreffende Handlung nicht nach gesetzlichen Vorschriften erlaubt ist, ist für die oben aufgeführten Weiterverwendungen des Materials die Einwilligung des jeweiligen Rechteinhabers einzuholen.

Weitere Details zur Lizenz entnehmen Sie bitte der Lizenzinformation auf http://creativecommons.org/ licenses/by/4.0/deed.de. 